Jan A. Plock Joachim Schmidt

Suzanne E. Anderson

Michael G. Sarr

Antoine Roggo

\section{Contrast-enhanced computed tomography in acute pancreatitis: does contrast medium worsen its course due to impaired microcirculation?}

Received: 7 July 2004

Accepted: 2 December 2004

Published online: 12 February 2005

(C) Springer-Verlag 2005

\begin{abstract}
Background: An early and accurate diagnosis of severe acute (necrotizing) pancreatitis is important to allow timely institution of therapy to limit the extra-pancreatic sequelae of this necrotizing process and to minimize the incidence of super-infection of the necrosis (i.e., progression to infected necrosis). Contrast-enhanced computed tomography (CECT) has become the cornerstone of diagnosis by confirming the clinical diagnosis of severe acute pancreatitis based on the various clinical scoring criteria.

Moreover, CECT serves as an anatomic roadmap for guiding radiological and surgical interventions. However, still-controversial experimental studies in animals in the mid-1990s suggested that the use of intravenous radiographic contrast media early in the course of the disease might exacerbate the necrotizing process by further impairing the already compromised pancreatic microcircu-
\end{abstract}

lation. A series of experimental and clinical studies followed that have both refuted and supported this claim; unfortunately, none is conclusive, and the topic remains, as yet, unresolved. Aims: Our objective was to review objectively the available literature found by a Medline search on this subject. Methods: Meta-analysis and review. Results and conclusion: Our conclusion, after analysis of these studies, is that there are no wellsubstantiated data that could resolve the controversy. However, several caveats will be offered.

Keywords Acute pancreatitis . Computed tomography $\cdot$ Contrast medium $\cdot$ Microcirculation

\section{Introduction}

Severe acute pancreatitis remains a devastating disease, the hallmark of which is the presence of necrosis of the pancreatic parenchyma and/or the peripancreatic retroperitoneal tissues. Early treatment within the initial hours of onset of symptoms in an intensive care unit utilizing aggressive hemodynamic resuscitation, nutritional support and, possibly, prophylactic antibiotics have served to decrease markedly the mortality over the past three decades [1-9]. The advent of a more objective means of imaging the necrosis (or more specifically imaging the lack of vascular perfusion) has contributed to this success. Similarly, different clinical and biochemical prognostic paradigms, such as $\mathrm{C}$ reactive protein, interleukins or procalcitonin, have been developed, not only to predict future complications, but, more importantly, to classify those patients at highest risk of developing potential complications, so that an aggressive therapeutic regimen can be instituted early in the course of the disease $[3,4,10-19]$. 


\section{Clinical assessment and classifications of acute pancreatitis}

Acute pancreatitis is a complex, frequent, and potentially life-threatening inflammatory disorder, which varies in spectrum from a mild to a severe clinical presentation. While the mild and moderate forms often have a self-limiting course, with negligible mortality, complications in the severe, fulminate form of this disease can occur, with development of the systemic inflammatory response syndrome (SIRS), pancreatic necrosis, infected necrosis, pancreatic abscess, hemorrhage, colonic necrosis, and pancreatic pseudocyst formation, many of which obligate a high mortality rate $[1,20]$. Infected necrosis and systemic sepsis with multiorgan failure may ensue, carrying a very high morbidity rate and a mortality rate of up to $40 \%[2,5,20-22]$.

Once the diagnosis of pancreatitis is suspected, the use of one of three current clinical classification systems will help to identify those patients at highest risk, i.e., that subset of severe acute (necrotizing) pancreatitis [5-9, 16, $23,24]$. Computed tomography (CT) is the most readily available and reliable imaging modality to assess suspected pancreatic disease [1, 2, 16, 23-28]. Intravenous contrastenhanced computed tomography (CECT) is accepted as the current gold standard for the verification and determination of the degree of the disease in the assessment of severe acute pancreatitis and for detecting and estimating the extent of pancreatic parenchymal necrosis [1, 16, 23-28]. Most surgeons and gastroenterologists seem to prefer the clinical staging systems (Ranson, Glasgow, APACHE II) for the initial assessment and reserve the CECT for patients with severe acute pancreatitis in an attempt to identify the presence of complications of the pancreatitis, such as infected necrosis, abscess or pseudocysts $[2,3,17-19$, 29-31] (Fig. 1).

The presence of $\geq 3$ Ranson [10] or Glasgow [11] criteria or $\geq 8$ points in the acute physiology and chronic health evaluation II criteria (APACHE II) [12] reliably identifies those patients with necrotizing pancreatitis with a sensitivity approaching $85 \%$. Although a small percentage of patients with documented necrotizing pancreatitis will have few, if any, of the clinical parameters used in these scoring systems [13, 14], the majority of patients with severe acute pancreatitis should be able to be recognized clinically, thereby allowing treatment to begin appropriately early in the course of the disease.

In mild forms of acute pancreatitis, an interstitial, edematous "pancreatitis" is present, characterized microscopically by marked edema of the interstitial space, with a minimal number of inflammatory cells present. Major necrosis of the pancreatic parenchyma is notably absent, although focal, microscopic acinar cell necrosis may arise $[15,26,32]$. Severe acute pancreatitis is associated with organ failure and/or local complications such as necrosis, abscess or pseudocysts. Intrapancreatic fat necrosis and acute inflammation represent the histological find-

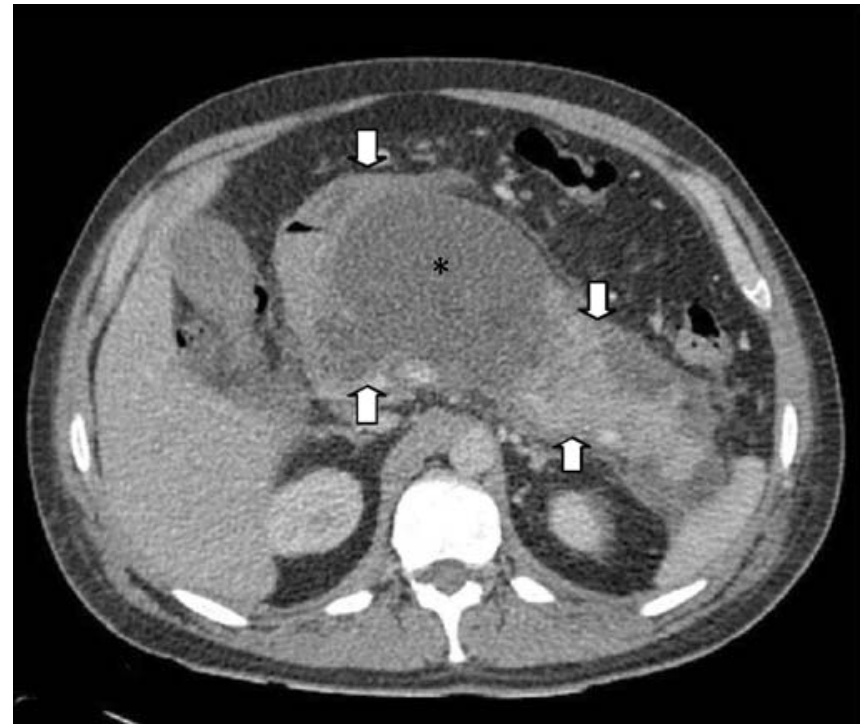

Fig. 1 CT scan with cystic $\left(^{*}\right)$ and necrotic alterations in patient with severe acute pancreatitis (arrows determine the extension of the pancreas)

ings, in addition to confluent zones of acinar cell necrosis $[15,26,32]$.

\section{Imaging modalities in necrotizing pancreatitis}

Numerous diagnostic imaging modalities are potentially available to detect the presence of acute pancreatitis, including transabdominal (or endoscopic) ultrasonography, endoscopic retrograde cholangiopancreatography (ERCP), $\mathrm{CT}$, and, more recently, magnetic resonance imaging (MRI). While all are effective in recognizing the presence of pancreatitis, their ability to detect the presence of tissue necrosis and the development of late complications of the pancreatitis vary markedly [16, 28, 33-35]. Recognition of the necrotizing component of severe acute pancreatitis (i.e., necrotizing pancreatitis) is very important for prognosis as well as for guiding therapy. Over the past 20 years CT has evolved as the most cost-effective and sensitive imaging modality, not only for confirming the clinical diagnosis of severe acute pancreatitis, but, more importantly, for predicting and staging the severity of the pancreatitis and for detecting the presence of serious complications $[1,2,16$, $23,24,26,35-38]$. The introduction and development of CECT permits recognition of viable (contrast-enhanced) and non-viable (non-enhancing) pancreatic parenchyma (Fig. 2).

CECT is optimally performed technically by taking the CT cuts of the peripancreatic area at the peak of pancreatic arterial perfusion, using a sufficiently high volume of iodinated contrast medium given as a rapid bolus infusion $[1,16,24,26]$. The reported sensitivity of CECT for the detection of necrosis in acute pancreatitis is $85 \%-92 \%$, 
Fig. 2 Axial magnetic resonance image (T2) with diffusely enlarged pancreas head (arrows) in acute pancreatitis

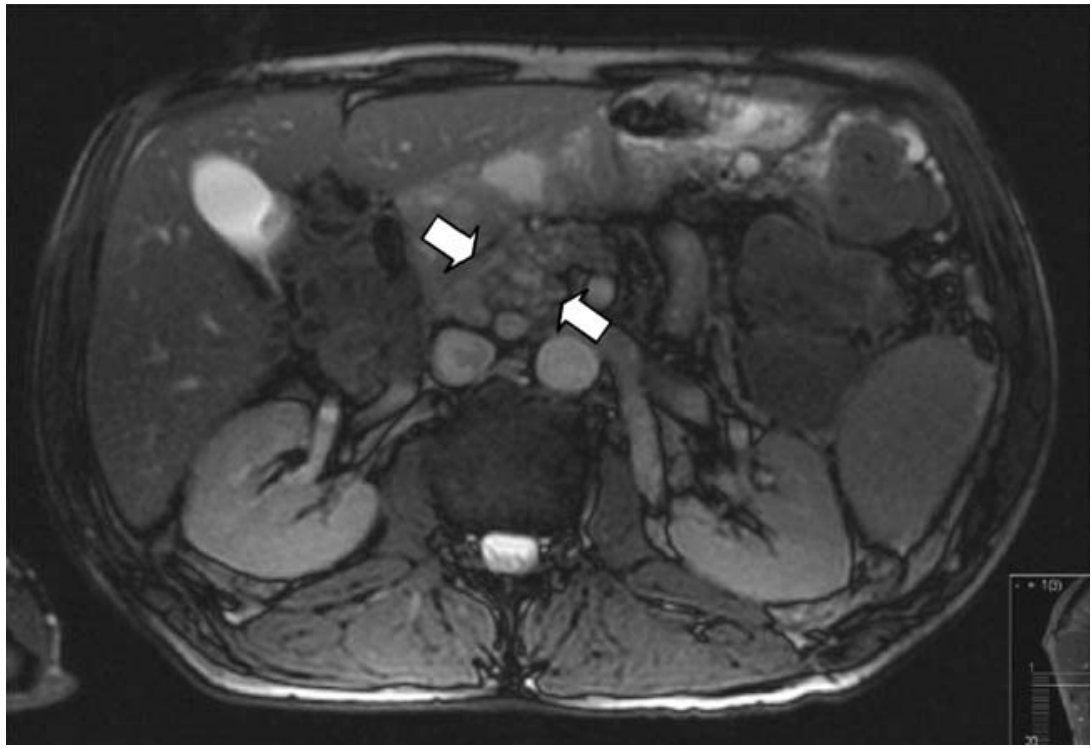

while the specificity of CECT has been shown to be $95 \%$ $100 \%[29,36,37]$. A positive correlation has been demonstrated between the prognostic signs, CT findings, and the clinical course. Balthazar and colleagues [23] classified the severity of findings on CECT appearance into five categories - A to E. Patients with pancreatitis of grades A-C usually manifest a mild, uncomplicated, clinical course, whereas grades $\mathrm{D}$ and $\mathrm{E}$ have a more prolonged course, with a higher morbidity rate, a higher incidence of pancreatic infection, and a higher mortality rate. According to this classification, the presence of necrosis and an acute inflammatory reaction are the two most important prognostic factors in the assessment of severity of acute pancreatitis. Using these two prognostic factors, Balthazar

Table 1 Calculation of CTSI [32]

\begin{tabular}{ll}
\hline Parameter Observation & Index \\
\hline
\end{tabular}

a) Inflammatory process

Grade A Normal pancreas

0

Grade B Focal or diffuse enlargement of pancreas 1

Grade C Pancreatic gland abnormalities associated 2 with peripancreatic inflammation

Grade D Fluid collection in a single location 3

Grade E Two or more fluid collections and/or the 4

b) Gland necrosis presence of gas in/or adjacent to pancreas

$\begin{array}{ll}\text { No necrosis } & 0 \\ <30 \% \text { necrosis } & 2 \\ 30-50 \% \text { necrosis } & 4 \\ >50 \% \text { necrosis } & 6\end{array}$

CTSI a) + b) $\quad$ Maximum 10 et al. [39] established a grading system (Table 1) called the CT severity index (CTSI); patients with a CTSI of $0-3$ had morbidity and mortality rates of $8 \%$ and $3 \%$, respectively, while patients with a CTSI of $7-10$ had rates of $92 \%$ and $17 \%$, respectively. The high specificity and sensitivity of CECT is, nevertheless, somewhat dependent on the time at which it is performed, as the complications seen in CECT and used for grading the severity of acute pancreatitis need time to develop.

\section{Contrast-enhanced computed tomography and microcirculation in acute pancreatitis-the controversy}

Marked reduction in the pancreatic microcirculation is an important mechanism in the pathophysiology of acute necrotizing pancreatitis, undoubtedly occurring in response to a variety of toxic, biologically active compounds [tumor necrosis factor- $\alpha(\mathrm{TNF} \alpha)$, interleukins, cytokines, etc.] released locally from the inflamed pancreas [40-47]. In a series of experimental studies using a rat model of acute pancreatitis [48-51], several investigators have provided evidence to suggest that the intravenous contrast media used for imaging of parenchymal enhancement in CECT aggravates the impairment of the pancreatic microcirculation during the early phases of acute pancreatitis. These experimental studies have sparked an ongoing controversy about the benefit or harm of CECT in the course of acute pancreatitis in humans and whether or not CECT is even necessary in the diagnosis of necrotizing pancreatitis in its early phase of acute pancreatitis $[1-4,14,16,19,23-27$, 29-31, 35, 37-39]. We will discuss the available clinical and experimental data on this topic chronologically. 


\section{Animal models}

In 1994, Foitzik et al. [48] studied an experimental model of graded acute pancreatitis in rats induced by intravenous cerulein followed by retrograde biliopancreatic ductal infusion of saline (mild acute pancreatitis) or differing amounts of glycodeoxycholic acid (moderate and severe acute pancreatitis). Animals with each severity of acute pancreatitis were divided into three groups to receive either intravenous $0.9 \%$ saline, or non-ionic, or ionic radiographic contrast material $7 \mathrm{~h}$ after initiation of acute pancreatitis. The animals were killed $24 \mathrm{~h}$ later, and the pancreata were analyzed microscopically. In one group receiving intravenous contrast agent, 20 of 36 animals (56\%) died during the first $24 \mathrm{~h}$ before the planned time for assessment. Because of the unexpected early mortality in this subgroup, the authors concluded that "the use of CECT to detect poorly perfused areas in the pancreas early in the course of acute severe pancreatitis may deserve to be reconsidered".

Later in 1994 and using the same animal model [49], those investigators further studied the topic by measuring hemoglobin content and oxygen saturation in pancreatic tissues in vivo, using diffuse reflectance spectrometry. The oxygen saturation of hemoglobin was reduced markedly in necrotizing pancreatitis in the rats given the intravenous contrast agent, suggesting that the radiological contrast agent impaired the pancreatic microcirculation in the necrotizing form of acute pancreatitis and, thereby, that "use of contrast-enhanced computed tomography early in human acute pancreatitis may promote the evolution of pancreatic necrosis".

In 1995 the third study from that group investigated pancreatic capillary blood flow, using intravital microscopy in the same rat model of acute necrotizing pancreatitis [50]. That study showed a marked decrease in total capillary blood flow in the pancreas in the group receiving the intravenous contrast agent. In addition, pancreatic vasomotion was analyzed in high-flow areas containing predominantly edematous changes as well as in low-flow areas with evolving necrosis. In the group receiving the contrast agent, capillary flow decreased significantly in the low-flow, but not in high-flow capillaries when compared to control animals. Those observations suggested that "radiographic contrast medium further impairs pancreatic capillary blood flow primarily in areas with pre-existing low-flow conditions and frequently converted poorly perfused regions to frank capillary stasis in experimental necrotizing pancreatitis".

That same year Hotz et al. [51] investigated the effect of hemodilution with dextran 70 versus Ringer's lactate on the pancreatic microcirculation in low-flow areas in rats receiving an intravenous contrast agent during acute necrotizing pancreatitis. Complete capillary stasis was greater in the capillaries of the Ringer's lactate group than in the dextran group ( $22 \pm 5 \%$ vs $2 \pm 1 \%$ of capillaries, $P<0.002)$. The investigators concluded that "while the mechanism of impaired pancreatic microcirculation induced by contrast medium is not yet clear, dextran seems to have a therapeutic effect on the specific impairment of pancreatic microcirculation during acute experimental pancreatitis".

Those studies, all in the same rat model of severe acute pancreatitis, suggested a potentially deleterious effect of iodinated radiographic contrast medium given during the early development of acute pancreatitis. In contrast, Kaiser and co-workers [52], in 1995 and using a different experimental model in the opossum, found contradictory results, complicating the issue. The group studied an opossum model of necrotizing pancreatitis induced by surgical ligation of the common bile/pancreatic duct. Beginning $48 \mathrm{~h}$ and $96 \mathrm{~h}$ later, intravenous contrast medium was infused into half the animals. When they were killed 3 days later, their serum amylase activity, pancreatic edema, acinar necrosis, and macroscopic/microscopic morphometric analysis of inflammation were similar in both groups. The authors concluded that "the concept that administration of contrast medium during early stages of pancreatitis is dangerous should not be accepted until additional experimental and clinical studies support its validity".

In 1998, Werner et al. [53] investigated the use of intravenous gadolinium-diethylenetriamine penta-acetic acid (GD-DTPA), an intravenous contrast agent used for MRI, using the same experimental rat model of Schmidt et al. [50]. They found no change in total capillary flow in the pancreas in the rats given intravenous GD-DPTA, as well as no changes in low-flow areas with evolving pancreatic necrosis compared to control rats given normal saline. Based on their two experimental studies, the group concluded that, in this rat model, use of GD-DPTA for MRI was safe in the early diagnosis of acute necrotizing pancreatitis when compared with the impairment of microcirculatory events induced by the intravenous contrast agent used in CT.

\section{Can the results in animal models be transferred to human acute pancreatitis?}

Those experimental studies had several limitations, especially when directly translating their results to the human scenario. First, in the study by Foitzik et al. [48], not all the animals were examined for histological changes, nor for the effects of the intravenous contrast agent. Similarly, the cause of death, the measure of outcome in this study, was not established nor reported. In addition, there was no control group of animals for the assessment of intravenous contrast agent alone. In the second study by Foitzik et al. [49] the authors failed to describe how many animals were examined, and they did not provide statistical justification for any difference between the two groups of animals concerning use of the intravenous control agent.

In human pancreatitis, acinar changes occur first, followed by progression of the necrotizing process to involve 
the vascular, ductal, and pancreatic/peripancreatic fat. These changes have been questioned by Balthazar and Freeny [25] in the rat model, but Rau et al. [54] were also able to demonstrate the early involvement of acinar cells in the course of acute pancreatitis. Balthazar and Freeny [25] also questioned the appropriateness of the timing of administration of intravenous contrast agent. Foitzik et al. $[48,49]$ infused the contrast agent $7 \mathrm{~h}$ after induction of pancreatitis, while in humans, CECT for acute pancreatitis is rarely performed earlier than $24 \mathrm{~h}$ after onset of the clinical symptoms. There were no data to support the hypothesis that the rats died because of increased necrosis after administration of intravenous contrast material. Nevertheless, the studies were performed carefully and in detail, with appropriate animal numbers, which enables a conclusion to be drawn, at least in this rat model.

Others argue that the model of Kaiser et al. [52], ligation of the common bile/pancreatic duct, more closely reflects the human situation (at least for pancreatitis). In this model there were no deleterious effects of intravenous contrast material when given 48-96 h after induction of pancreatitis, a time more consistent with the timing of CECT used in the clinical situation. However, we must acknowledge the marked differences between the studies. While many more animals have been studied with the use of the original rat model of Foitzik et al. [48] versus that of the opossum model of Kaiser et al. [52], it remains unknown which model is most appropriate for humans. In addition, the timepoints of administration of contrast medium were different: $7 \mathrm{~h}$ after induction of acute pancreatitis in Foitzik's study and $48 \mathrm{~h}$ and $96 \mathrm{~h}$ in Kaiser's study. Kaiser's animals were additionally pretreated with antibiotics for 1 week for prevention of skin infection, with the last dose on the day of operation, which was an attempt to reproduce prophylactic antibiotics for severe acute pancreatitis. The conclusions drawn from the multiple studies in the rat model were to avoid early CECT in the course of severe acute pancreatitis, if no contradictions exist. The study by Kaiser et al. [52] in the opossum model found no adverse effects of intravenous contrast medium, when given at somewhat later times after onset of acute pancreatitis and, of course, in a different animal model.

\section{Results from clinical studies of early CECT}

Inspired by the controversial findings in 1996, McMenamin and Gates [55] reported a retrospective clinical analysis of the effect of CECT on the course of 57 patients with acute pancreatitis. The 31 patients who underwent CECT appeared to have a longer duration of clinical acute pancreatitis than the 26 patients not undergoing CECT ( $11 \pm 2$ vs $6 \pm 1$ days, $P=0.004$ ), despite their having similar APACHEII scores at admission. This uncontrolled, retrospective review suggested that the use of intravenous contrast during CT might be deleterious. Another clinical study by
Carmona-Sanchez et al. in 2000 [56] reviewed the effect of intravenous contrast agents on the course and outcome of 126 patients with clinically mild acute pancreatitis, 52 of whom underwent CECT. In the CECT group, six of 52 patients developed local or systemic complications compared to only one of the 74 patients who did not undergo CECT $(P<0.02)$. In addition, median hospital stay was longer (18.4 vs 11.4 days; $P<0.01$ ), leading these authors to conclude, "Although other factors cannot be excluded, such as severity not adequately identified, this association (increased incidence of local and/or systemic complications in the CECT group) suggests a potentially harmful effect of intravenous contrast material." The primary criticism of those two retrospective studies revolve around their retrospective design and the question of comparability of the groups compared. Obviously, one group was subjected to CECT, while the other group was not, raising the question of presumed severity of the pancreatitis in the CECT group (Fig. 3).

In contrast, Hwang et al. [57] conducted a randomized, prospective trial in 20 patients with severe acute pancreatitis, comparing outcome in patients undergoing $\mathrm{CT}$ with or without intravenous contrast agent on the first day of admission. No significant differences between groups were detected in numerous laboratory parameters of pancreatitis, including serum amylase and lipase activities, nor in concentrations of serum C-reactive protein, creatinine, and calcium when evaluated at multiple time points during hospitalization. Overall morbidity, duration of hospital stay, and mortality also showed no significant differences. The authors concluded, "these data may support us in allowing patients with acute pancreatitis to undergo an abdominal CT examination with enhancing contrast medium." However, conclusions from that study are somewhat limited compared to the other clinical studies, because the number of patients included seemed to be small. Unfortunately, no follow-up (CE)CT was performed.

In 2002 Uhl et al. [58] reported a retrospective, reanalysis of 302 patients entered initially into a randomized, double-blind, multicenter trial of the use of octreotide in patients with moderate to severe acute pancreatitis; 264 patients underwent CECT after onset of symptoms, while 38 patients did not. In 189 patients, CECT was performed during their hospital course. Octreotide proved to be of no benefit. Although patients in the CECT group at admission had more severe acute pancreatitis, as indicated by a higher C-reactive protein and APACHE-II score than the patients without CECT, the early $(<14$ days) mortality rate was less $(3.8 \%$ vs $15.8 \%, P<0.002)$ as was the 1 -month mortality rate $(6.4 \%$ vs $15.8 \%, P<0.04)$. Systemic and local complications (shock, sepsis, fluid collections) occurred with similar frequency in both groups. The authors concluded that their findings "could contribute to change the clinical practice in approaching patients with severe acute pancreatitis". Any negative effects of the intravenous contrast agent should have been detected, because CECT was per- 
Fig. 3 Post-intravenous contrast-enhanced magnetic resonance image (T1) with multiple, dilated, pancreatic ducts (arrows) in the pancreatic head

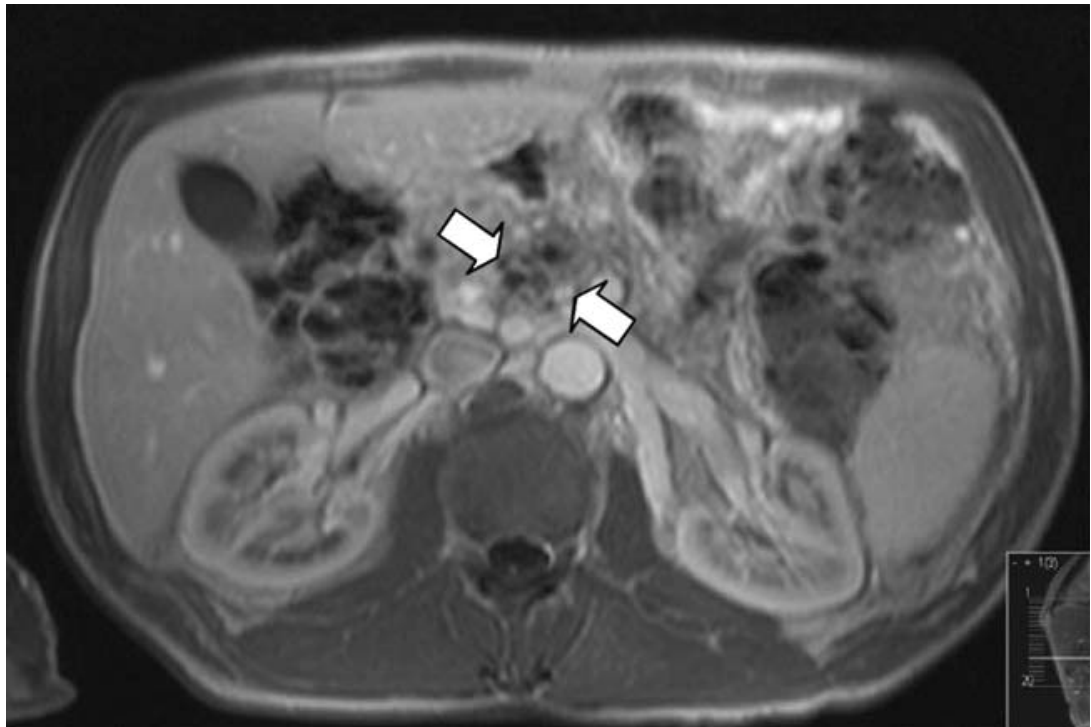

formed within $96 \mathrm{~h}$ of the onset of clinical symptoms. No deleterious effects on the clinical course or radiological findings were detected secondary to the administration of the intravenous contrast medium. Indeed, repetitive use of CECT seemed to reduce mortality by increasing the detection of potential complications requiring the need for, and best timing of, surgical therapy. However, that study, like those by McMenamin and Gates [55] and CarmonaSanchez and colleagues [56] is subject to the same criticism of a retrospective analysis.

In 1999, Wang et al. [59] stated that, in their opinion, there is an increased alteration of the microcirculation in vivo after administration of intravenous radiographic contrast agent in acute pancreatitis and that this might contribute to the progression of severity of pancreatitis. Those authors suggested that low osmolar, non-ionic, radiographic contrast medium would be less vasoactive than ionic contrast and, therefore, should be used preferentially in the assessment of acute pancreatitis. This contention was not supported by the findings of Foitzik et al. [48], which suggested that non-ionic agents did not provide any benefit over ionic contrast agents. In the microvascular system, the radiographic agents primarily seem to cause vasodilatation, with the exception of the kidney where they lead to vasoconstriction $[60,61]$.

The last important studies of this topic utilized magnetic resonance technology in acute pancreatitis. Hirota et al. [33] reported that pancreatic necrosis could be imaged by gadolinium-enhanced MRI as well as by CECT. In their prospective study, MRI even proved superior to CECT in differentiating regions of pancreatic necrosis from perinecrotic fluid collections and in visualization of heterogeneity within the pancreatic necrosis. Similar findings were reported by Zhang et al. in 2003 [34] and by Arvanitakis et al. in 2004 [62]. Those authors also addressed important logistic problems, e.g., the use of MRI in severely ill patients in the intensive care unit, the higher costs of MRI versus $\mathrm{CT}$, and the availability of MRI scanners for emergency and routine use in the community. An increasing number of reports support a role for MRI in the diagnosis and assessment of acute pancreatitis [63-67]. However, a cautionary note is pertinent. Two case reports in the literature suggest that acute pancreatitis may occur after administration of gadodamide (Omniscan, Nycomed, Ireland). The letter in Lancet 1999 by Terzi and Sokmen [68], and its clarification by Tsushima and Endo in 2000 [69], as well as the report in 2001 by Schenker et al. [70], are important, because the current literature and specific studies of this topic in both animals and humans are lacking. We could not find any reports suggesting that gadopentate (Magnevist, Schering) induces acute pancreatitis.

\section{Conclusions}

What are we to learn from this experience? First, much of this discussion about the potential harm of intravenous contrast material used during CECT is currently a moot point, because CECT is usually not used (or needed) in making the clinical diagnosis of severe acute pancreatitis in the first 48-72 h of onset; the clinical scoring systems are sufficient for the majority of patients with severe acute pancreatitis. Treatment in an intensive care unit setting should begin immediately and be based on the clinical diagnosis of severe acute pancreatitis, and treatment should not be withheld despite the lack of an "objective" diagnosis based on an objective imaging modality. In this setting, CECT is best reserved for recognition of complications of the necrotizing process that usually begin more than 5 days after onset of the pancreatitis, i.e., infected necrosis, pancreatic abscess, extensive peripancreatic fluid collections, or later pseudocysts. In contrast, in the small subset of patients in 
whom the diagnosis is in doubt or in whom an abdominal catastrophe requiring urgent operation is feared, CECT should not necessarily be summarily excluded for fear of exacerbating the pancreatitis. Indeed, the evidence of a harmful effect of the intravenous contrast agents used during CECT is not convincing, especially if the patient is resuscitated appropriately from a hemodynamic standpoint. For those institutions with ready access to MRI, this im- aging modality allows another option with use of intravenous gadopentate, a non-iodinated contrast agent. However, until MRI becomes more financially appropriate and universally available, CECT should remain the "gold standard" when necessary for the diagnosis of necrotizing pancreatitis and, especially, as the primary imaging modality for identifying and directing interventional and surgical therapy of complications of necrotizing pancreatitis.

\section{References}

1. Balthazar EJ (1994) Pancreatitis. In: Gore RM, Levine MS, Laufer I (eds) Textbook of gastrointestinal radiology. Saunders, Philadelphia, pp 2132-2160

2. Buechler MW, Uhl W, Friess H, Malfertheiner P (1999) Acute pancreatitis, novel concepts in biology and therapy. Blackwell Science, Berlin

3. Mayumi T, Ura H, Arata S, Kitamura N, Kiriyama I, Shibuya K, Sekimoto M, Naga N, Hirota M, Yoshida M, Ito Y, Hirata K, Takada T (2002) Evidencebased clinical practice guidelines for acute pancreatitis: proposals. J Hepatobiliary Pancreat Surg 9:413-422

4. Ogawa M, Hirota M, Hayakawa T, Matsuno S, Watanabe S, Atomi Y, Otsuki M, Kashima K, Koizumi M, Harada H, Yamamoto M, Nishimori I (2002) Development and use of a new staging system for severe acute pancreatitis based on a nationwide survey in Japan. Pancreas 25:325-330

5. Bradley EL (1989) Antibiotics in acute pancreatitis. Current status and future directions. Am J Surg 158:472-478

6. Ratschko M, Fenner T, Lankisch PG (1999) The role of antibiotic prophylaxis in the treatment of acute pancreatitis. Gastroenterol Clin North Am 28:641-659

7. Delcenserie R, Yzet T, Ducroix JP (1996) Prophylactic antibiotics in the treatment of acute pancreatitis. Pancreas 13:198-201

8. Sainio V, Kemppainen E, Puolakkainen P, Taavitsainen M, Kivisaari L, Valtonen V, Haapiainen R, Schroder T, Kivilaakso E (1995) Early antibiotic treatment in acute necrotising pancreatitis. Lancet 346:663-667

9. Schwarz M, Isenmann R, Meyer H, Beger HG (1997) Antibiotika bei nekrotisierender Pankreatitis, Ergebnisse einer kontrollierten Studie. Dtsch Med Wochenschr 122:356-361

10. Ranson J (1982) Etiological and prognostic factors in human acute pancreatitis: a review. Am J Gastroenterol 9:633-638

11. Blamey SL, Imrie CW, O'Neill J, Gilmour WH, Carter DL (1984) Prognostic factors in acute pancreatitis. Gut 25:1340-1346
12. Knaus WA, Draper EA, Wagner DP, Zimmerman JE (1985) APACHE II: a severity of disease classification system. Crit Care Med 13:818-829

13. Corfield AP, Cooper MJ, Williamson $\mathrm{RCN}$, Mayer AD, McMahon MJ, Dickson AP, Shearer MG, Imrie CW (1985) Prediction of severity in acute pancreatitis: prospective comparison of three prognostic indices. Lancet 2:403407

14. Block S, Maier W, Bittner R, Buechler MW, Malfertheiner P, Beger HG (1986) Identification of pancreas necrosis in severe acute pancreatitis. Imaging procedures versus clinical staging. Gut 27:1035-1042

15. Bradley EL III (1993) A clinically based classification system for acute pancreatitis. Arch Surg 128:586-590

16. Balthazar EJ (2002) Staging of acute pancreatitis. Radiol Clin North Am 40:1199-1209

17. Birgisson H, Moller PH, Birgisson S, Thoroddsen A, Asgeirsson KS, Sigurjonsson SV, Magnusson J (2002) Acute pancreatitis: a prospective study of its incidence, aetiology, severity, and mortality in Iceland. Eur J Surg 168:278-282

18. King NK, Powell JJ, Redhead D, Siriwardena AK (2003) A simplified method for computed tomographic estimation of prognosis in acute pancreatitis. Scand J Gastroenterol 38:433436

19. Lankisch PG, Blum T, Maisonneuve P, Lowenfels AB (2003) Severe acute pancreatitis: when to be concerned? Pancreatology 3:102-110

20. Silverstein W, Isikoff MB, Hill MC, Barkin J (1981) Diagnostic imaging of acute pancreatitis: prospective study, using CT and sonography. Am J Radiol 137:497-502

21. Schmid SW, Uhl W, Friess $H$, Malfertheiner P, Buechler MW (1999) The role of infection in acute pancreatitis. Gut 45:311-316

22. Beger HG, Bittner R, Block S, Buchler M (1986) Bacterial contamination of pancreatic necrosis. A prospective clinical study. Gastroenterology 91:433-438
23. Balthazar EJ, Ranson CHC, Naidich DP, Megibow AJ, Caccalave R, Cooper MW (1985) Acute pancreatitis: prognostic value of CT. Radiology 156:767-772

24. Balthazar EJ (2002) Acute pancreatitis: assessment of severity with clinical and CT evaluation. Radiology 223:603-613

25. Balthazar EJ, Freeny PC (1994) Contrast-enhanced computer tomography in acute pancreatitis: is it beneficial or harmful? Gastroenterology 106:259 262

26. Balthazar EJ, Freeny PC, van Sonnenberg E (1994) Imaging and intervention in acute pancreatitis. Radiology 193:297-306

27. Balthazar EJ (1989) CT diagnosis and staging of acute pancreatitis. Radiol Clin North Am 27:19-37

28. Ly JN, Miller FH (2002) MR imaging of the pancreas: a practical approach. Radiol Clin North Am 40:1289-1306

29. Johnson CD, Stephens DH, Sarr MG (1991) CT of acute pancreatitis. Correlation between lack of contrast enhancement and pancreatic necrosis. Am J Roentgenol 156:93-95

30. Lankisch PG, Struckmann K, Assmus C, Lehnick D, Maisonneuve P, Loewenfels AB (2001) Do we need a computed tomography examination in all patients with acute pancreatitis within $72 \mathrm{~h}$ after admission to hospital for the detection of pancreatic necrosis? Scand J Gastroenterol 36:432-436

31. Dervenis C, Johnson CD, Bassi C, Bradley E, Imrie CW, McMahon MJ, Modlin I (1999) Diagnosis, objective assessment of severity, and management of acute pancreatitis. Int J Pancreatol 25:195-210

32. Banks PA (1994) A new classification system for acute pancreatitis. Am J Gastroenterol 89:151-152

33. Hirota M, Kimura Y, Ishiko T, Beppu T, Yamashita Y, Ogawa M (2002) Visualization of the heterogeneous internal structure of so-called "pancreatic necrosis" by magnetic resonance imaging in acute necrotizing pancreatitis. Pancreas 25:63-67 
34. Zhang XM, Shi H, Parker L, Dohke M, Holland GA, Mitchell DG (2003) Suspected early or mild chronic pancreatitis: enhancement patterns on gadolinium chelate dynamic MRI. J Magn Reson Imaging 17:86-94

35. Balthazar EJ (2002) Complications of acute pancreatitis: clinical and CT evaluation. Radiol Clin North Am 40:1211-1227

36. Kemppainen E, Puolakkainen P, Leppäniemi A, Hietaranta A, Groenroos J, Haapiainen R (1998) Diagnosis of acute pancreatitis. Ann Chir Gynaecol 87:191-194

37. Balthazar EJ (1989) CT diagnosis and staging of acute pancreatitis. Radiol Clin North Am 27:19-37

38. London NJM, Neoptolemos JP, Lavelle J, Bailey I, James D (1989) Contrastenhanced abdominal computed tomography scanning and prediction of severity of acute pancreatitis: a prospective study. Br J Surg 76:268-272

39. Balthazar EJ, Robinson D, Megibow A, Ranson J (1990) Acute pancreatitis: value of CT in establishing prognosis. Radiology 174:331-336

40. Bassi D, Kollias N, Fernandez-del Castillo C, Foitzik T, Warshaw AL, Rattner DW (1994) Impairment of pancreatic microcirculation correlates with the severity of acute experimental pancreatitis. J Am Coll Surg 179:257263

41. Huch K, Schmidt J, Schratt W, Sinn HP, Buhr H, Herfarth C, Klar E (1995) Hyperoncotic dextran and systemic aprotinin in necrotizing rodent pancreatitis. Scand J Gastroenterol 30:812816

42. Klar E, Mail G, Messmer K, Herfarth C, Rattner D, Warshaw AL (1993) Improvement of impaired pancreatic microcirculation by isovolemic hemodilution protects pancreatic morphology in acute biliary pancreatitis. Surg Gynecol Obstet 176:144-150

43. Klar E, Messmer K, Warshaw AL, Herfarth C (1990) Pancreatic ischaemia in experimental acute pancreatitis: mechanism, significance and therapy. Br J Surg 77:1205-1210

44. Gross V, Leser HG, Heinisch A, Scholmerich J (1993) Inflammatory mediators and cytokines: new aspects of the pathophysiology and assessment of severity of acute pancreatitis. Hepatogastroenterology 40:522-531

45. Denham W, Norman J (1999) The potential role of therapeutic cytokine manipulation in acute pancreatitis. Surg Clin North Am 79:767-781

46. Beger HG, Rau B, Mayer J, Pralle U (1997) Natural course of acute pancreatitis. World J Surg 21:130-135
47. Uhl W, Buchler M, Malfertheiner P, Isenmann $\mathrm{R}$, Martini $\mathrm{M}$, Beger $\mathrm{HG}$ (1991) Pancreatic necrosis develops within four days after the acute attack. Gastroenterology 100:A302

48. Foitzik T, Bassi DG, Schmidt J (1994) Intravenous contrast medium accentuates the severity of acute necrotizing pancreatitis in the rat. Gastroenterology 106:207-214

49. Foitzik T, Bassi DG, Fernandez-del Castillo C, Warshaw AL, Rattner DW (1994) Intravenous contrast medium impairs oxygenation of the pancreas in acute necrotizing pancreatitis in the rat. Arch Surg 129:706-711

50. Schmidt J, Hotz HG, Foitzik T (1995) Intravenous contrast medium aggravates the impairment of pancreatic microcirculation in necrotizing pancreatitis in the rat. Ann Surg 221:1-8

51. Hotz HG, Schmidt J, Ryschich EW, Foitzik T, Buhr HJ, Warshaw AL, Herfarth C, Klar E (1995) Isovolemic hemodilution with dextran prevents contrast medium induced impairment of pancreatic microcirculation in necrotizing pancreatitis of the rat. Am J Surg 169:161-166

52. Kaiser AM, Grady T, Gerdis T, Saluja M, Steer ML (1995) Intravenous contrast medium does not increase the severity of acute necrotizing pancreatitis in the opossum. Dig Dis Sci 40:1547-1553

53. Werner J, Schmidt J, Warshaw AL, Gebhard MM, Herfarth C, Klar E (1998) The relative safety of MRI contrast agent in acute necrotizing pancreatitis. Ann Surg 227:105-111

54. Rau B, Bauer A, Wang A, Gansauge F, Weidenbach $\mathrm{H}$, Nevalainen T, Poch B, Beger HG, Nussler AK (2001) Modulation of endogenous nitric oxide synthase in experimental acute pancreatitis: role of anti-ICAM-1 and oxygen free radical scavengers. Ann Surg 233:195203

55. McMenamin DA, Gates LK Jr (1996) A retrospective analysis of the effect of contrast-enhanced CT on the outcome of acute pancreatitis. Am J Gastroenterol 91:1384-1387

56. Carmona-Sanchez R, Uscanga L, Bezaury-Rivas P, Robles-Diaz G, Suazo-Barahona J, Vargas-Vorackova F (2000) Potential harmful effect of iodinated intravenous contrast medium on the clinical course of mild acute pancreatitis. Arch Surg 135:1280-1284

57. Hwang TL, Chang KY, Ho YP (2000) Contrast-enhanced dynamic computed tomography does not aggravate the clinical severity of patients with severe acute pancreatitis: reevaluation of the effect of intravenous contrast medium on the severity of acute pancreatitis. Arch Surg 135:287-290
58. Uhl W, Roggo A, Kirschstein T, Anghelacopoulos SE, Gloor B, Mueller CA, Malfertheiner P, Buechler MW (2002) Influence of contrast-enhanced computed tomography on course and outcome in patients with acute pancreatitis. Pancreas 24:191-197

59. Wang YX, Chen S, Morcos SK (1999) Contrast-enhanced CT in acute pancreatitis. Br J Radiol 72:1029

60. Dawson P (1989) Cardiovascular effects of contrast agents. Am J Cardiol 64:2E-9E

61. El Sayed AA, Haylor JL, El Nahas AM, Salzano S, Morcos SK (1991) Haemodynamic effects of water-soluble contrast media on the isolated perfused rat kidney. Br J Radiol 64:435-439

62. Arvanitakis M, Delhaye M, De Maertelaere V, Bali M, Winant C, Coppens E, Jeanmart J, Zalcman M, Van Gansbeke D, Deviere H, Matos C (2004) Computed tomography and magnetic resonance imaging in the assessment of acute pancreatitis. Gastroenterology 126:715-723

63. Piironen A (2001) Severe acute pancreatitis: contrast-enhanced CT and MRI features. Abdom Imaging 26:225233

64. Amano Y, Oishi T, Takahashi M, Kumazaki T (2001) Nonenhanced magnetic resonance imaging of mild acute pancreatitis. Abdom Imaging 26:59-63

65. Ward J, Chalmers AG, Guthrie AJ, Larvin M, Robinson PJ (1997) T2weighted and dynamic enhanced MRI in acute pancreatitis: comparison with contrast enhanced CT. Clin Radiol 52:109-114

66. Elmas N (2001) The role of diagnostic radiology in pancreatitis. Eur J Radiol 38:120-132

67. Freeny PC (1999) Pancreatic imaging. New modalities. Gastroenterol Clin North Am 28:723-746

68. Terzi C, Sokmen S (1999) Acute pancreatitis induced by magnetic-resonance-imaging contrast. Lancet 354:1789-1790

69. Tsushima Y, Endo K (2000) Contrast agent confusion. Lancet 355:654

70. Schenker MP, Solomon JA, Roberts DA (2001) Gadolinium arteriography complicated by acute pancreatitis and acute renal failure. J Vasc Interv Radiol 12:393 